Monatsschrift f. Geburtshülfe u. Gynäkologie 1919;49:I-IV

\title{
Contents, Vol. 49, 1919
}

\section{Inhalts - Verzeichnis.}

Seite

Original arbeiten.

Arndt, W., ÜberdasphysiologischeundpathologischeVor-

kommen morphologisch darstellbarer Lipoide in den Ge-

schlechtsorganen des Weibes 315, 413

Dietrich, H. A., Zur Bevölkerungspolitik 428

Foges, A. u.K. Hofßtätter, Sieben Jahre Hypo-

physenmedikation in der praktischen Geburtshülfc. Be-

merkungen zu der im Heft 5, Bd. XLVITI, erschienenen

. Arbeit von Prof. J. Hofbauer207

Prank, G. v., Die subkutane Symphysiotoïnie bei Eri³/st-

gebärenden 332

G a 11 , P., Beckenerweiternde Operationen oder transperi-

tonealer Kaiserschnitt? 438

Gänßle, H. F., Klinische Erfahrungen an 138 Fallen von

komplettem Dammriß 243

G»rdlund, W., Über die Atiologie und Therapie bei

Kraurosis vulvae 106

G erich, O., Die Appendicitis catarrhalis chronica in ihren

Beziehungen zur Perimetritis 303

Gustafsson, L., Ein Fall von Chorionepitheliom, ent-

standen während der Gravidität. (Hierzu Taf. 1) . . 75 H e y m a n, J., Ein Fall von

Spontangeburt eines reifen

Kindes in Stirnlage mit nach hinten gerichteter Stirn. 234 Hirsch, M., Frauenheilkunde und Bevölkerungspolitik . 200 Hofstätter, R., über Befunde'bei hyper hy pop hysiert en

Tieren 387

K r i t z 1 e r, Ein Fall von Selbstabreißung der Scheide bei

Sohräglage nebst Bemerkungen über die Ursache ihres

verhältnismäßig seltenen Vorkommens und über etwaige

Verhütung 84

Krumma cher, A., Die Bebandlung der prof usen Menses

mit Strytysatum. (Vorläufige Mitteilung) 260

Kuncz, A., Ein Fall von ausgetragener Tuboovarial-

Schwangerschaft 168

L i n d i g, P., Temperatursteigerungen beim Neugeborenen

im Lichte serologischer Forschung 348

Mayer, A·, Über die blutstillende Wirkung des Follikel-

saftes ; ... 228 
Momm u. Kraemer, Die Muttermilch zur Kriegszeit 91 Ottow, B., Wie ist die Dilatation der Harnröhre bei

Seheidenmangel zu deuten und über den sogen. Coitus

urethralis $\quad 174$

$-\mathrm{IV}-$

Seite

Palm, H., Zur Frage der Entstehung des Kernikterus der

Neugeborenen 264

Schaef er, A·, Die Autoinfusion in der Geburtshülfe . 162 Schroder, R. u. A. L·oeser, Die

Trichomonaden-

kolpitis. ïhre K, linik und Bakteriologie 23

$\mathrm{S}$ e it $\mathrm{z}, \mathrm{L}$. u. H. W i nt $\mathrm{z}$, Über die Beziehungen des

Corpus luteum zur Menstruation 1

Trebing, J., Lokale und interne Anvvendung des Jod in

der Gynäkologie 44\#5

Werner, E., Ein Fall von Encephalocele occipitalis mit

postoperativem, jedoch spontan geheiltem Hydrocephalus 98 Zw $\beta i f \beta 1$, E., Über Narkophin,

Laudanon, Mannit-Scopo-

lamiu und Pantopon-Atropinschvvefelsäure 38

2.ix den Tagesfragen.

L i e s k e, H., Die ärztliche Überwachung der Hebamme . 46

Berichte.

Hüssy, P., Bericht über die italienische gynäkologische

und gebartshülfliche Literatur 1914-1917 55

Mandach, G· v., Bericht über die französische Literatur

$1917 \quad 122$

Richard Werth† 129

Friedrich Schauta $\uparrow$ (mit Porträt) ....... 151

Bernhard Sigmund Schultze- Jena † . . . 377

Vereins- und Literaturbeilage.

Gynäkologische Gesellschaft inBreslau- Sitzung v. 14. I. 1919. 280 Münchener gynäkologisohe Gesellschaft. Sitzung vom 14. u.

28. II. 1918209

Geburtshülflich-gynäkologische Gesellschaft in Wien. Sitzung

vom 10. XII. 1918 u. 14. I. $1919 \quad$ 287, 366

Geburtshülflich - gynäkologische Mitteilungen aus Medizini-

schenGesellschaftenundVereinen68, 138, 220, 292, 372,451

Buchanzeigen 69, 139, 221, 293

Literatur-Verzeiehnis 72, 149, 225, 300, 373;

452

Tagesnachriehten 137,450

Berichtigung 208, 450

Personalien 67, 137, 279, 376

Sachregister $\quad 456$

Namenregister $\quad 459$ 